\title{
SOME ROUGHNESS RESULTS CONCERNING REDUCIBILITY FOR LINEAR DIFFERENCE EQUATIONS
}

\section{GARYFALOS PAPASCHINOPOULOS}

\author{
Democritus University of Thrace \\ School of Engineering \\ 67100 , Xanthi, Greece
}

(Received October 20, 1937)

ABSTRACT. In this paper we prove first that the exponential dichotomy of linear difference equations is "rough". Horeover we prove that if the coefficlent matrix of a linear difference equation is almost periodic, then the joint property of having an exponential dichotomy with a projection $P$ and being reducible with $P$ by an almost periodic kinematics similarity is "rough".

KEY WORDS AND PHRASES. Exponential dichotomy, almost periodic matrix, adjoint equation. 1980 AMS SUBJECT CLASSIFICATION CODE. Primary 39A10, Secondary 39A11.

1. INTRODUCTION. Consider the difference equation

$$
x(n+1)=A(n) x(n)
$$

where $A(n)$ is a $k \times k$ invertible matrix function for $n \in Z=\{, \ldots,-1,0,1, \ldots\}$.

In what follows we denote by $|x|$ the Euclidean norm of the vector $x$. Then the induced norm $|A|$ for a matrix $A$ is the square root of the largest eigenvalue of ÁA (A is the transpose of $A$ ).

The difference equation (1.1) is said to possess an exponential dichotomy on $Z$ if there exist a projection $P\left(P^{2}=P\right)$ and constants $k \geqslant 1, a>0$ such that

$$
\begin{aligned}
& \left|X(n) P X^{-1}(m)\right|<K e^{-a(n-m)}, n>m \\
& \left|X(n)(I-P) X^{-1}(m)\right|<K e^{-a(m-n)}, m>n
\end{aligned}
$$

where $X(n)$ is a fundamental matrix solutions of (1.1) and $n, m \varepsilon Z$. In this situation we also say that $X(n)$ has an exponential dichotomy with projection $P$ and constants $K, a$. 
Let $\bar{A}(n)$ be $a k \times k$ invertible matrix funtion. Consider the equation

$$
y(n+1)=\vec{A}(n) y(n)
$$

Equations (1.1) and (1.3) are said to be kinematically similar if there exists a $k \times k$ invertible matrix function $S(n)$ which is bounded together with its inverse, such that the change of variables $x(n)=S(n) y(n)$ transforms (1.1) into (1.3).

Equation $(1.1)$ is said to be reducible with projection $P$ if it is kinematically similar to $(1.3)$ where $\overline{P A}(n)=\bar{A}(n) P$.

A matrix function $A(n)$ is almost periodic if from every sequence $\left\{a_{m}\right\}, a_{m} \in Z$ there exists a subsequence $\left\{a_{m}^{\prime}\right\}$ such that $\lim A\left(n+a_{n}^{\prime}\right), m \rightarrow \infty$ exists uniformly with respect to $n$.

According to Palmer [1, p.377] equation (1.1) has the property (H) if it has a fundamental matrix solution $x(n)$ which satisfies (1.2) and, moreover, is reductble with $P$ by a kinematic similarity $S(n)$ such that

1) $S(n)$ is almost periodic and

ii) the reduced system has a fundamental matrix solution which commutes with $P$ and has an exponential dichotomy with projection $P$.

The main results of this paper are the following:

i) Suppose that $(1.1)$ has a fundamental matrix solution $X(n)$ which satisfies (1.2). Let $B(n)$ be a matrix such that $A(n)+B(n)$ is invertible and sup $|B(n)|$, $n \in Z\}=\delta, \delta$ is sufficiently small. Then the perturbed equation

$$
y(n+1)=(A(n)+B(n)) y(n)
$$

has a fundamental matrix solution which also has an exponential dichotomy with the same projection $P$.

ii) Let $A(n)$ be an almost periodic matrix function. Suppose that (1.1) has property $(H)$. Let $B(n)$ be an almost periodic matrix function such that $A(n)+B(n)$ is invertible and $\sup \{|B(n)|, n \varepsilon z\}=\delta, \delta$ is sufficiently small. Then the perturbed equation (1.4) also has property $(H)$.

We note that these results are the discrete analogues of those of Coppel [2], [3] and Palmer [1] but the passage from the continuous case to the discrete case is not at all straightforward. We also note that a roughness theorem for dichotomies of difference equations has been proved by Henry (see Theorem 7.6.7 [4, p. 232]) using admissibility theory. However, we can easily prove that Proposition 1 of this paper applies to dichotomies on an arbitrary set $I=\left\{m_{1}, m_{1}+1, \ldots, m_{2}\right\}, m_{1} m_{2} \varepsilon Z$. Moreover, some arguments of Proposition 1 are used in the proof of Proposition 2.

It is worthwhile to study linear difference equations because recently there has been an increase in interest in discrete dynamical systems. It is know that linear difference equations arise as variational equations along the orbits of such systems. 
Some results on exponential dichotomy, reducibility and almost periodicity of difference equations are included in the papers [4], [5], [6], [7], [8], [9], [10], $[11],[12]$.

2. MAIN RESULTS.

First we derive some results for equations of the form

$$
\Delta x(n)=c(n) x(n+1), n \in Z
$$

where $\Delta x(n)=x(n+1)-x(n)$ and $C(n)$ is a $k x$ matrix function such that $I-C(n)$ is invertible. Since $I-C(n)$ is invertible, equation (2.1) has a fundamental matrix solution $X(n)$.

It will be assumed that all the definitions of this paper for equations of the form (1.1) are valid also for equations of the form (2.1).

The following lemma shows that uniform asymptotic stability is preserved under small perturbations of the coefficient matrix for the equations of the form (2.1).

LEMMA 1. Suppose that (2.1) has a fundamental matrix solution $X(n)$ such that

$$
\left|X(n) X^{-1}(m)\right|<K e^{a(n-m)}, n>m, a \varepsilon R
$$

Let $\wedge(n)$ be a $k \times k$ matrix function such that the matrix function $I-(C(n)+\wedge(n))$ is invertible and

$$
\sup \{|\wedge(s)|, s \varepsilon z\}=\delta, \delta>0
$$

Then the perturbed equation

$$
\Delta y(n)=(C(n)+\Lambda(n)) y(n+1)
$$

has a fundamental matrix solution $Y(n)$ which satisfies

$$
\left|\mathrm{Y}(\mathrm{n}) \mathrm{y}^{-1}(\mathrm{~m})\right|<\mathrm{Ke} \mathrm{b}^{\mathrm{(n-m})}, \mathrm{n}>\mathrm{m}
$$

where $b=a+\delta \mathrm{Ke}^{\mathrm{a}}$.

PROOF. It 18 easy to show that every solution $y(n)$ of (2.4) satisfies

$$
y(n)=x(n) x^{-1}(m) y(m)+\sum_{s=m+1}^{n} x(n) x^{-1}(s-1) \wedge(s-1) y(s) .
$$

Then from (1), (6) and using the discrete Gronwal1's lemma [13, p. 337] 1f $w(n)=e^{-a n}|y(n)|$ we obtain

$$
w(n)<K e^{\delta k e^{a(n-m)}} w(m) .
$$


So

$$
|y(n)|<K e^{b(n-m)}|y(m)|
$$

Thus the proof of the lemma is completed.

REMARK 1. It is easy to show that the lemma holds if instead of (2.1) and (2.4) we have equations $(1.1)$ and (1.4) correspondingly. The only change is that the constant $b$ is equal to $a+\delta \mathrm{Ke}^{-\mathrm{a}}$.

LEMMA 2. Suppose that (2.1) has an exponential dichotomy (1.2) where $P$ is orthogonal $(\stackrel{*}{\mathrm{P}}=\mathrm{P})$. Then $(2.1)$ is reducible to

$$
\Delta y(n)=D(n) y(n+1)
$$

where the coefficient matrix $D(n)$ commutes with $P$ and (2.5) has a fundamental matrix solution $Y(n)$ which commutes with $P$ and satisfies

$$
\begin{aligned}
& \left|Y(n) P Y^{-1}(m)\right|<2 K^{2} e^{-a(n-m)}, n>m \\
& \left|Y(n)(I-P) Y^{-1}(m)\right|<2 K^{2} e^{-a(m-n)}, m>n .
\end{aligned}
$$

Also the matrix transformation $T(n)$ satisfies

$$
|\mathrm{T}(\mathrm{n})|<2^{1 / 2},\left|\mathrm{~T}^{-1}(\mathrm{n})\right|<2^{1 / 2} \mathrm{~K}, \mathrm{n} \varepsilon \mathrm{Z} \text {. }
$$

PROOF. From Lemma 1 [3, p. 39] there exists a matrix function $T(n)$ such that

$$
T(n) P T^{-1}(n)=X(n) P X^{-1}(n)
$$

and from $(1.2)$

$$
|T(n)|<2^{1 / 2},\left|T^{-1}(n)\right|<2^{1 / 2} K, n \in Z
$$

Also from Lemma 1 [3, p. 39] $T(n)=X(n) R^{-1}(n)$, where $R(n)$ commutes with P. The change of variables $x(n)=T(n) y(n)$ transforms (2.1) into (2.5) where $D(n)=T^{-1}(n) C(n) T(n+1)-T^{-1}(n) \Delta T(n)$. We have that $R(n)=T^{-1}(n) X(n)$ is a fundamental matrix solution of $(2.5)$. Therefore $D(n)=(\Delta R(n)) R^{-1}(n+1)$. Hence $D(n)$ commutes with P. If $Y(n)=R(n)$ from (1.2) and (2.7) we have that the inequalities (2.6) are satisfied and the proof of the lemma is completed.

In Proposition 1 below since every projection $P$ of rank equal to $\ell$ is similar to the projection $Q=d i a g ~ Q=d i a g\left(I_{\ell}, 0\right)$, I is the identity $\ell x \ell$ matrix (see [3, p. 4142]), we may suppose that $P=d i a g\left(I_{\ell}, 0\right)$.

The following lemma shows that exponential dichotomy is preserved under small perturbations of the coefficient matrix for the equations of the form (2.1). 
LEMMA 3. Suppose that (2.1) has a fundamental matrix soluion $X(n)$ which satisifes (1.2) with $\mathrm{P}=\operatorname{diag}\left(I_{\ell}, 0\right)$. Let $\Lambda(n)$ be a matrix such that the $I-(C(n)+\Lambda(n))$ is invertible. Then if

$$
\sup \{|\wedge(n)|, n \in Z\}=\delta<\min \left\{\frac{1-e^{-2 a}}{72 K^{5} e^{-a}}, \frac{a}{6 K^{3} e^{a}}\right\},
$$

the perturbed equation (2.4) has a fundamental matrix solution $Y(n)$ which satisfies

$$
\begin{aligned}
& \left|Y(n) P Y^{-1}(m)\right|<12 K^{3} e^{-v(n-m)}, n>m \\
& \left|Y(n)(I-P) Y^{-1}(m)\right| * 12 K^{3} e^{-v(m-n)}, m>n
\end{aligned}
$$

where $v=a-6 K^{3} \delta e^{a}$.

PROOF. We apply the change of variables $x(n)=T(n) y(n)$ as in Lemma 2 to equations $(2.1)$ and (2.4). Then (2.1) and (2.4) are kinematically similar to (2.5) and

$$
\Delta w(n)=(D(n)+M(n)) w(n+1)
$$

respectively where $D(n)=T^{-1}(n) C(n) T(n+1)-T^{-1}(n) \Delta T(n)$ and $M(n)=T^{-1}(n) \wedge(n) T(n+1)$.

For any matrix A we put

$$
E_{1}=P E P+(I-P) E(I-P) \text { and } E_{2}=P E(I-P)+(I-P) E P \text {. }
$$

Then we have $E=E_{1}+E_{2}$. Obviously $E_{1}$ commutes with $P$.

We claim that there exists an invertible matrix function $s_{1}(n)$ which is bounded together with its inverse such that the change of variables $w(n)=S_{1}(n) v(n)$ transforms (2.10) into the system

$$
\Delta v(n)=\left(D(n)+\left\{M(n) s_{1}(n+1)\right\}_{1}\right) v(n+1)
$$

It is easy to show that the claim is true if $S_{1}(n)$ satisfies the equation

$$
\Delta S_{1}(n)=D(n) S_{1}(n+1)-S_{1}(n) D(n)+M(n) S_{1}(n+1)-S_{1}(n)\left\{M(n) S_{1}(n+1)\right\}
$$

or putting $S_{1}(n)=I+H(n)$ if $H(n)$ satisfies

$$
\Delta H(n)=D(n) H(n+1)-H(n) D(n)+\{M(n)(I+H(n+1))\}_{2}-H(n)\{M(n)(I+H(n+1))\}_{1}
$$

We must prove that (2.13) has a bounded solution. Consider the space $E$ of all matrix functions $H(n)$ such that $|H(n)|<\frac{1}{2}$, nEZ. Define the operator $\bar{T}$ on $E$ as follows:

$$
\overline{\mathrm{TH}}(\mathrm{n})=\sum_{s=-\infty}^{\mathrm{n}-1} \mathrm{Y}(\mathrm{n}) \mathrm{PY}^{-1}(\mathrm{~s})(\mathrm{I}-\mathrm{H}(\mathrm{s})) \mathrm{M}(\mathrm{s})(\mathrm{I}+\mathrm{H}(\mathrm{s}+1)) \mathrm{Y}(\mathrm{s}+1)(\mathrm{I}-\mathrm{P}) \mathrm{Y}^{-1}(\mathrm{n})-
$$


$-\sum_{s=n}^{\infty} Y(n)(I-P) Y^{-1}(s)(I-H(s)) M(s)(I+H(s+1)) Y(s+1) P Y^{-1}(n)$

where $Y(n)$ is the fundamental matrix solution of (2.5) which satisfies (2.6).

First we prove that $\bar{T} H$ is in E. From (2.6) we get

$$
|\bar{T} H(n)|<\frac{36 K^{5} \delta e^{-a}}{1-e^{-2 a}}, n \varepsilon Z
$$

From (2.8) we have that $\overline{\mathrm{T}} \mathrm{H}$ is in $\mathrm{E}$.

We prove now that $\bar{T}: E \rightarrow E$ is a contraction. Consider $H, G \in E$.

It is easy to prove that

$(I-H(s)) M(s)(I+H(s+1))-(I-G(s)) M(s)(I+G(s+1))=M(s)(H(s+1)-G(s+1))-(H(s)-G(s)) M(s)-$ $H(s) M(s)(H(s+1)-G(s+1))-(H(s)-G(s)) M(s) G(s+1)$.

Then it is easy to prove

$$
|\bar{T} H(n)-\bar{T} G(n)|<\frac{48 K^{5} \delta e^{-a}}{1-e^{-2 a}}|H-G|,
$$

where $|H-G|=s u p\{|H(n)-G(n)| n \varepsilon Z\}$. So $\bar{T}$ is a contraction on the Banach Space E. So there exists a unique $H \varepsilon E$ such that $\bar{T} H(n)=H(n), n \varepsilon Z$.

We prove now that $H(n)$ is a solution of (2.13). We have

$$
\begin{aligned}
\Delta H(n)= & \left(\Delta\left(Y(n) \wedge_{1}(n)\right)\right) Y^{-1}(n+1)+Y(n) \wedge_{1}(n) \Delta Y^{-1}(n)- \\
& -\left(\Delta\left(Y(n) \wedge_{2}(n)\right)\right) Y^{-1}(n+1)-Y(n) \wedge_{2}(n) \Delta Y^{-1}(n)
\end{aligned}
$$

where

$$
\begin{aligned}
& \wedge_{1}(n)=\sum_{s=-\infty}^{n-1} P Y^{-1}(s)(I-H(s)) M(s)(I+H(s+1)) Y(s+1)(I-P) \\
& \Lambda_{2}(n)=\sum_{s=n}^{\infty}(I-P) Y^{-1}(s)(I-H(s)) M(s)(I+H(s+1)) Y(s+1) P .
\end{aligned}
$$

Since $\Delta Y(n)=D(n) Y(n+1)$ we get $D Y^{-1}(n)=-Y^{-1}(n) D(n)$. Hence

$$
\begin{aligned}
\Delta H(n) & =D(n) Y(n+1) \wedge_{1}(n+1) Y^{-1}(n+1)+Y(n)\left(\Delta_{1}(n)\right) Y^{-1}(n+1)- \\
& -Y(N) \wedge_{1}(n) Y^{-1}(n) D(n)-D(n)-D(n) Y(n+1) \wedge_{2}(n+1) Y^{-1}(n+1)- \\
& -Y(n)\left(\Delta \wedge_{2}(n)\right) Y^{-1}(n+1)+Y(n) \wedge_{2}(n) Y^{-1}(n) D(n) .
\end{aligned}
$$


Since from Lemma 2, $Y(n)$ commutes with $P$ we have

$$
\Delta H(n)=D(n) H(n+1)-H(n) D(n)+\{(I-H(n)) M(n)(I+H(n+1))\}_{2} \cdot
$$

It holds

$P H P=0$ and $(I-P) H(I-P)=0$, so $H=H P+P H$.

Therefore we can easily prove

$$
\{(I-H(n)) M(n)(I+H(n+1))\}_{2}=\{M(n)(I+H(n+1))\}_{2}-H(n)\{M(n)(I+H(n+1))\}_{1} .
$$

So $H(n)$ is a solution of (2.13) such that $|H(n)|<\frac{1}{2}$, neZ. Therefore $S_{1}(n)=I+H(n)$ satisfies (2.12) and $\left|s_{1}(n)\right|<\frac{3}{2},\left|s_{1}^{-1}(n)\right|<2$. Hence our claim 1s true. So $(2.10)$ and $(2.11)$ are kinematically similar. It holds

$$
\left|\left\{M(s) S_{1}(s+1)\right\}_{1}\right|<\left|M(s) s_{1}(s+1)\right|<3 K \delta
$$

Since the coefficient matrices of (2.5) and (2.11) commute with $P$ each equation decomposes into two equations. Suppose that (2.5) decomposes into the equations

$$
\begin{aligned}
& \Delta y_{1}(n)=D_{1}(n) y_{1}(n+1) \\
& \Delta y_{2}(n)=D_{2}(n) y_{2}(n+1)
\end{aligned}
$$

where (2.15) is uniform asymptotically stable. Suppose that (2.11) decomposes into the equations

$$
\begin{aligned}
& \Delta v_{1}(n)=\left(D_{1}(n)+\left\{M(n) S_{1}(n+1)\right]_{11}\right) v_{1}(n+1) \\
& \Delta v_{2}(n)=\left(D_{1}(n)+\left\{M(n) S_{1}(n+1)\right\}_{12}\right) v_{2}(n+1) .
\end{aligned}
$$

Applying Lemma 1 to (2.15) and (2.17) and using (2.8) and (2.14) there exists a fundamental matrix solution $v_{1}(n)$ of $(2.17)$ such that

$$
\left|v_{1}(n) v_{1}^{-1}(m)\right|<2 k^{2} e^{-v(n-m)}, n>m
$$

To prove an analogous result for (2.18) we use the same method as in [6, p. 69]. Consider the equations

$$
\begin{aligned}
& y_{2}(n+1)=\left(I-\stackrel{\star}{D}_{2}(n)\right) y_{2}(n) \\
& v_{2}(n+1)=\left(I-\stackrel{\star}{D}_{2}(n)-\left\{M(n){ }^{\star} S_{1}(n+1)\right\}_{12}\right) v_{2}(n)
\end{aligned}
$$


Since for a matrix A it holds $|\stackrel{*}{A}|=|A|$ equation (2.16) has a fundamental matrix solution $Y_{2}(n)$ such that

$$
\left|\stackrel{*}{Y}_{2}^{-1}(m) \stackrel{*}{Y}_{2}(n)\right|=\left|Y_{2}(n) Y_{2}^{-1}(m)\right|<2 k^{2} e^{-a(m-n)}, m>n \text {. }
$$

Since $\stackrel{\star}{\mathrm{Y}}_{2}^{-1}(\mathrm{n})$ is a fundamental matrix solution of (2.19) and applying Remark 1 to (2.19) and (2.20) there exists a fundamental matrix solution $\vec{v}_{2}(n)$ of (2.20) which satisifies

$$
\left|\tilde{v}_{2}(m) \tilde{v}_{2}^{-l}(n)\right|=\left|\tilde{v}_{2}^{-1}(n) \stackrel{*}{\tilde{v}_{2}}(m)\right|<2 k^{2} e^{-v(m-n)} \cdot m>n .
$$

But $v_{2}(n)=\stackrel{\star}{v}^{-1}(n)$ is a fundamental matrix solution of $(2.18)$. So (2.11) has a funamental matrix solution $V(n)=\operatorname{diag}\left(v_{1}(n), V_{2}(n)\right)$ which has an exponential dichotomy with $P$ and constants $2 \mathrm{~K}^{2}, \mathrm{v}$. Hence $(2.10)$ has a fundamental matrix solution $W(n)=S_{1}(n) V(n)$ which has an exponential dichotomy with $P$ and constants $6 K^{2}, v$. Thus $Y(n)=T(n) S_{1}(n) V(n)$ is a fundamental matrix solution of (2.4) which satisfies (2.9) and the proof of the lemma is completed.

To prove our main results we use the following lemma.

LEMMA 4. Suppose that (1.1) has an exponential dichotomy (1.2) where $P=d i a g\left(I_{\ell}, 0\right)$.

Then the adjoint equation of $(1.1)$

$$
x(n+1)=A^{*-1}(n) x(n)
$$

has a fundamental matrix solution which has an exponential dichotomy with projection $I-P$ and constants $K, a$.

PROOF. Suppose that (1.1) has a fundamental matrix solution $X(n)$ which satisfies (1.2). Then since we use the Euclidean norm we obtain

$$
\begin{aligned}
& \left|\mathrm{X}^{*-1}(\mathrm{~m})(I-P) \stackrel{*}{\mathrm{X}}(\mathrm{n})\right|<\mathrm{Ke}-\mathrm{a}(\mathrm{m}-\mathrm{n}), \mathrm{m}>\mathrm{n} \\
& \left|\mathrm{X}^{\star-1}(\mathrm{~m}) \mathrm{P}^{\star} \mathrm{X}(\mathrm{n})\right|<\mathrm{Ke}^{-\mathrm{a}(\mathrm{n}-\mathrm{m})}, \mathrm{n}>\mathrm{m} .
\end{aligned}
$$

Since $Y(n)=\stackrel{X}{X}^{-1}(n)$ is a fundamental matrix solution of (2.21) the proof of the lemma is completed.

PROPOSITION 1. Suppose that (1.1) has an exponential dichotomy (1.2) where $P=\operatorname{diag}\left(I_{\ell}, 0\right)$. Let $B(n)$ be a matrix function such that the $\operatorname{matrix} A(n)+B(n)$ is invertible. Suppose that $B(n)$ satisfies

$$
\sup \{|B(n)|, n \varepsilon Z]=\delta<\min \left\{\frac{1-e^{-2 a}}{72 k^{5} e^{-a}}, \frac{a}{6 K^{3} e^{a}}\right\}
$$

Then the perturbed equation (1.4) has a fundamental matrix solution which has an exponential dichotomy with the projection $P$ and constants $12 \mathrm{~K}^{3}, \mathrm{v}$. 
PROOF. Equation $(2.21)$ can be written to the form

$$
\Delta x(n)=(I-\stackrel{*}{A}(n)) x(n+1)
$$

Consider equation

$$
\Delta y(n)=(I-\stackrel{*}{A}(n) \stackrel{*}{B}(n)) y(n+1)
$$

From Lemma 4 we have that (2.23) has a fundamental matrix solution which has an exponential dichotomy with the projection I-P and constants K,a. Since (2.22) holds, from Lemma 3 we have that $(2.24)$ has a fundamental matrix solution which has an exponential dichotomy with the projection I-P and constants $12 \mathrm{~K}^{3}, \mathrm{v}$. But (2.24) can be written as the adjoint equation of (1.4). Then from Lemma 4 the proof of the proposition is completed.

To prove the second roughness result we use two lemmas.

LEMMA 5. Suppose that (2.1) has a fundamental matrix solution $X(n)$ which has an exponential dichotomy with a projection $P$ and that (2.1) is reducible withP by the kinematic similarity $S(n)$ to (2.5). If $Y(n)$ is a fundamental matrix solution of (2.5) and $Q$ is a projection the following statements are equivalent:

1) $Y(n)$ commutes with $Q$ and $Y(n)$ has an exponential dichotomy with $Q$.

1i) $X(n) P X^{-1}(n)=S(n) Q S^{-1}(n), n \in Z$.

The proof of the lemma is analogous to the proof of Lemma $2[1, \mathrm{p} .376]$.

REMARK 2. Using Lemma 5 we can easily prove the following:

Suppose that (2.1) has property (H) (See introduction for definition). Let $X(n)$ be the fundamental matrix of (2.1), $P$ the projection and $S(n)$ the kinematic similarity which are involved in (H). Then the condition (i1) of (H) is equivalent to $X(n) P X^{-1}(n)=S(n) P S^{-1}(n), n \varepsilon Z$.

REMARK 3. Using the same argument as in [1, p.377] we can prove that if $S(n)$ is an almost periodic invertible matrix with bounded inverse then $\mathrm{s}^{-1}(\mathrm{n})$ is also almost periodic.

LEMMA 6. Let $C(n)$ be an almost periodic matrix function on $Z$. Suppose that (2.1) has property (H). Let $\wedge(n)$ be an almost periodic matrix unction such that $I-(C(n)+\Lambda(n))$ is invertible. Then if $\Lambda(n)$ satisfies $(2.8)$, the perturbed equation (2.4) has property $H$.

PROOF. Consider the fundamental matrix solution $X(n)$ of $(2.1)$, the projection $P$ and the kinematic similarity $S(n)$ which are involved in $(H)$.

There exists an invertible matrix $L$ such that $P=L Q L^{-1}, Q=d i a g\left(I_{\ell}, 0\right)$. Using the same argument as in the proof of Lemma 3 [1, p. 378] we can prove tha there exists a matrix function $T(n)=S(n) L R^{-1}(n), R(n)$ is an almost periodic matrix such that the change of variables $x(n)=T(n) y(n)$ transforms (2.1) into (2.5) where $Q D(n)=D(n) Q$, $n \in Z$ and (2.5) into (2.10). We also have $T(n)$ satisfies (2.7) and (2.5) has a fundamental matrix solution $Y(n)=T^{-1}(n) X(n) L$ which commutes with $Q$ and has an exponential dichotomy with the projection $Q$ and constants $2 \mathrm{~K}^{2}$,a. Arguing as in Lemma 
3 equation (2.13) has a unique bounded solution $H(n),|H(n)|<\frac{1}{2}$, nez such that for $\mathrm{m} \varepsilon \mathrm{Z}$ we get

$$
\begin{aligned}
& H(n+m)-H(n)=\sum_{S=-\infty}^{n-1}\left(Y(n+m) Q Y^{-1}(s+m)-Y(n) Q Y^{-1}(s)\right) F(s+m) Y(s+1+m)(I-Q) Y^{-1}(n+m) \\
& +Y(n) Q Y^{-1}(s)(F(s+m)-F(s)) Y(s+1+m)(I-Q) Y^{-1}(n+m)+ \\
& +Y(n) Q Y^{-1}(s) F(s)\left(Y(s+1+m)(I-Q) Y^{-1}(n+m)-Y(s+1)(I-Q) Y^{-1}(n)\right) \\
& -\sum_{s=n}^{\infty} \ldots \ldots .
\end{aligned}
$$

where $F(s)=(I-H(s)) M(s)(I+H(s+1))$. We have that $N(n)=Y(n+m) Q Y^{-1}(s+m)$ is a solution of

$$
\Delta N(n)=D(n) N(n+1)+(D(n+m)-D(n)) N(n+1)
$$

Then from the variation of constants formula which is taken by using the same method as in [14, $.11-12]$ and since $Y(n), D(n)$ commute with $Q$ we get for $n>s$

$$
\begin{aligned}
& \left.N(n)=Y(n) Y^{-1}(s) N(s)+\sum_{u=s+1}^{n} Y(n) Y^{-1}(u-1)(D(u+m-1)-D(u-1)) N(u)\right)= \\
& =Y(N) Q Y^{-1}(s)+\sum_{u=s+1}^{n} Y(n) Q Y^{-1}(u-1)(D(u+m-1)-D(u-1)) N(u) .
\end{aligned}
$$

Then we obtain

$$
\left|Y(n+m) Q Y^{-1}(s+M)-Y(n) Q Y^{-1}(s)\right|<\frac{4 K^{4}}{e^{8}-1}\left|D_{m}-D\right|
$$

where $\left|D_{m}-D\right|=\sup \{|D(n+m)-D(n)|, n \varepsilon Z\}$. Using the same argument we can easily prove

$$
\left|Y(s+1+m)(I-Q) Y^{-1}(n+m)-Y(s+1)(I-Q) Y^{-1}(n)\right|<\frac{4 K^{4} e^{a}}{e^{a}-1}\left|D_{m}-D\right| \text {. }
$$

$$
\begin{aligned}
& \text { Since from the proof of } \quad \text { Lemma } 3 \quad M(n)=T^{-1}(n) \wedge(n) T(n+1) \text { we get } \\
& |M|=\sup \{|M(n)|, n \in Z\}<2 K 5 \text {. Using }(2.25),(2.26),(2.27) \text { and }(2.8) \text { we obtain }
\end{aligned}
$$

$$
\left|H_{m}-H\right|<\frac{54 e^{a_{K} K^{4}}\left|M_{m}-M\right|}{e^{2 a}-1}+\frac{216 K^{6}|M| e^{a}\left|D_{m}-D\right|}{\left(e^{a}-1\right)^{2}}
$$

Since from Remark $3, T^{-1}(n)$ is almost periodic, we have that $M(n), D(n)$ are also almost periodic matrix functions. Hence from (2.28) $H(n)$ is almost periodic. From Lemma 3 we have that the perturbed equation (2.4) has a fundamental matrix solution which has an expoenential dichotomy with the projection Q. Then as in the proof of Lemma 3 the transformation $y(n)=T(n)(I+H(n)) v(n)$ transforms (2.4) into (2.11) which 
has a fundamental matrix solution which commutes with $Q$ and has an exponential dichotomy with the projection Q. Using Lemma 5 we have

$$
Y(n) P Y^{-1}(n)=T(n)(I+H(n)) L^{-1} P\left(T(n)(I+H(n)) L^{-1}\right)^{-1}
$$

Using Remark 2 we have that (2.4) has property (H) with the projection $P$ and Kinematic similarity $T(n)\left(I+H(n) L^{-1}\right.$ Thus the proof of the lemma is completed.

PROPOSITION 2. Let $A(n)$ be an almost periodic invertible matrix function. Suppose that (1.1) has property (H) with the projection $P$. Consider an almost periodic matrix function $B(n)$ such that $A(n)+B(n)$ is invertible for neZ. Then if $B(n)$ satisfies $(2.22)$ we have that the perturbed equation (1.4) also has property (H) with the same projection $P$.

PROOF. We use the same argument as in Proposition 1. Since (1.1) has property (H) from Remark 2 we have

$$
X(n) P^{-1}(n)=S(n) P^{-1}(n)
$$

where $X(n)$ is the fundamental matrix solution of (1.1), P the projection and $S(n)$ the kinematic similarity involved in $(H)$. Then we obtain

$$
\stackrel{\star *}{X}^{-1}(n)(I-\stackrel{*}{\mathrm{P}}) \stackrel{\star}{\mathrm{X}}(\mathrm{n})=\stackrel{*}{\mathrm{~S}}-1(\mathrm{n})(I-\stackrel{*}{\mathrm{P}}) \stackrel{\star}{\mathrm{S}}(\mathrm{n})
$$

It is obvious that the matrices $\stackrel{\star}{S}(n), \stackrel{\star}{S}-1(n)$ are almost periodic since $s(n), S^{-1}(n)$ are almost periodic.

From Lemma $4 \hbar^{-1}(n)$ is a fundamental matrix of (2.23) which has an exponential dichotomy with I-ث. So from Remark 2 we have that (2.23) has property (H) with the projection $I-\stackrel{*}{P}$. Since $B(n)$ satisfies (2.22) from Lemma 6 we have that (2.24) also has property $(H)$ with the projection $I-\stackrel{*}{P}$. Let $Y(n)$ be the fundamental matrix solution of $(2.24)$ and $S_{2}(n)$ the kinematic similarity involved in (H). From Remark 2 we have

$$
\mathrm{Y}(\mathrm{n})(\mathrm{I}-\stackrel{\star}{\mathrm{P}}) \mathrm{Y}^{-1}(\mathrm{n})=\mathrm{S}_{2}(\mathrm{n})(\mathrm{I}-\stackrel{\star}{\mathrm{P}}) \mathrm{S}_{2}^{-1}(\mathrm{n}) \text {, so } \mathrm{Y}(\mathrm{n}) \stackrel{\star}{\mathrm{PY}}-1(\mathrm{n})=\mathrm{S}_{2}(\mathrm{n}) \stackrel{\star}{\mathrm{PS}} \mathrm{S}_{2}^{-1}(\mathrm{n})
$$

Then we get

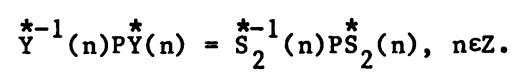

From Lemma $4, \stackrel{\star}{\mathrm{Y}}-1(\mathrm{n})$ is a fundamental matrix solution of (1.4) which has an exponential dichotomy with projection P. So from Remark 2 we have that (1.4) has property (H) with the projection $P$. Thus the proof of the proposition is completed.

REMARK 4. Using the same argument as in [1, p. 382] we can easily prove that when $A(n)$ is a real almost peirodic $2 \times 2$ matrix such that (1.1) has an exponential dichotomy then (1.1) has property (h). 
ACKNOWLEDGMENT. The author would like to thank the refree for his helpful suggestions.

\section{REFERENCES}

1. PALMER, K.J. On the reducibility of almost periodic systems of linear differential equations, J. Differential Equations 36, 374-390 (1980).

2. COPPEL, W.A. Dichotomies and reducibility, J. Differential Equations 3 (1967), 500-521.

3. COPPEL, W.A. Dichotomies in stability theory, Lecture Notes in Mathematics, No. 629, Springer-Verlag, Berlin, 1978.

4. HALANAY, A. Solutions periodiques et presque-periodiques des systems d'equations aux differences finies, Arch. Rational Mech. Anal. 12 (1963), 134-149.

5. PAPASCHINOPOULOS, G. and SCHINAS, A. Criteria for an exponential dichotomy of difference equations, zechoslovak Math. J. 35 (110), (1985) p. 295-299.

6. PAPASCHINOPOULOS, G. and SCHINAS, J. A criterion for the exponential dichotomy of difference equations, Rend Sem. Fac. Sci. Univ. Cagliari, 54, fasc 1 (1984), 61-71.

7. PAPASCHINOPOULOS, G. and SCHINAS, J. Multiplicative separation, diaonalizability and structural stability of linear difference equations, Differential Equations: Qualitative theory (Szeged 1984), Collog. Math. Soc Janos Bolyai 47, North-Holland, Amsterdam-New York.

8. PAPASCHINOPOULOS, G. Exponential separation, exponential dichotomy and almost peridocitdy of linear difference equations, J. Math. Anal. App1. 120, (1986) 276-287.

9. PAPASCHINOPOULOS, G. and SCHINAS, J. Structural stability via the density of a class of linear discrete systems, J. Math. Ana1. Appl. 127, (1987), 530-539.

10. PAPASCHINOPOULOS, G. A characterization of exponential separation in terms of reducibility for linear difference equations, J. Math. Anal. Appl., to appear.

11. SCHINAS, J. and PAPASCHINOPOULOS, G. Topological equivalence for linear discrete systems via dichotomies and Lyapunov functions, Bol1. Un. Math. Ital. 6, 4 (1985), 61-70.

12. SLYUSARCHUK, V.E. Exponential dichotomy for solutions of discrete systems, Ukranian Math. J. 35, (1983), 98-103.

13. SCHINAS, J. Stability and conditional stability of time-dependent difference equations in Banach spaces, J. Inst Maths. Applic. (1974) 14, 335-346.

14. MILLER, K.S. Linear difference equations, Benjamin, New York, 1968.

15. HENRY, D. Geometric theory of semilinear parabolic equations, Lecture Notes in Mathematics, No. 840, Springer Verlag, Berlin 1981. 


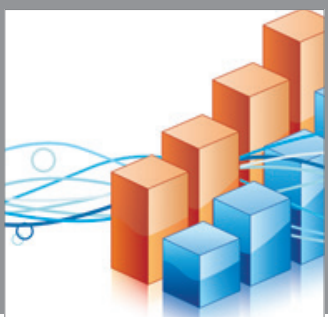

Advances in

Operations Research

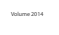

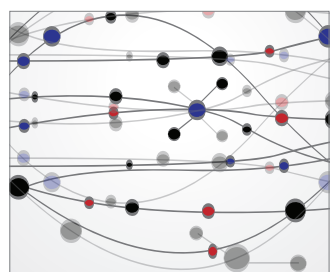

\section{The Scientific} World Journal
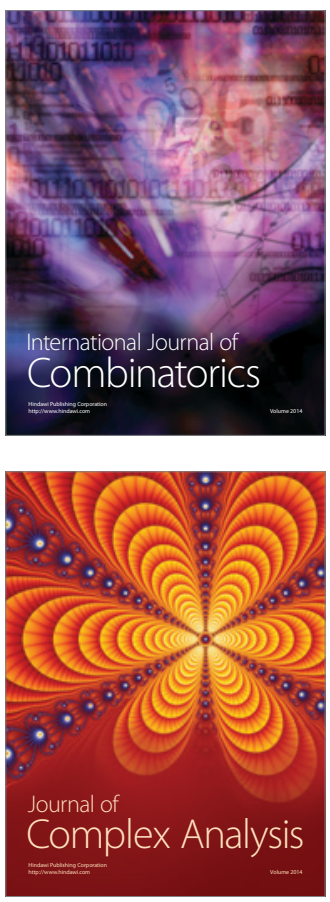

International Journal of

Mathematics and

Mathematical

Sciences
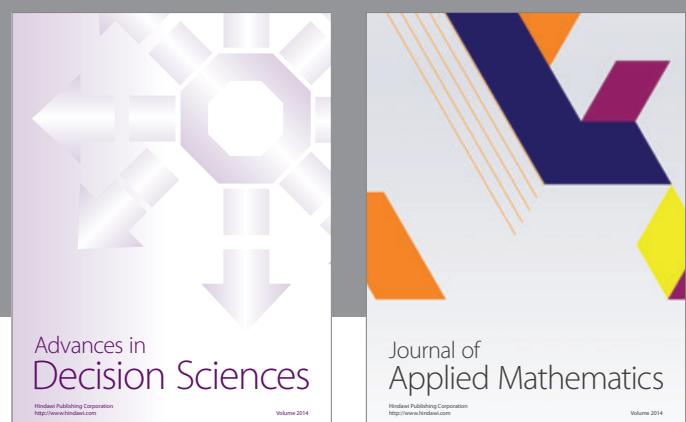

Journal of

Applied Mathematics


Submit your manuscripts at http://www.hindawi.com
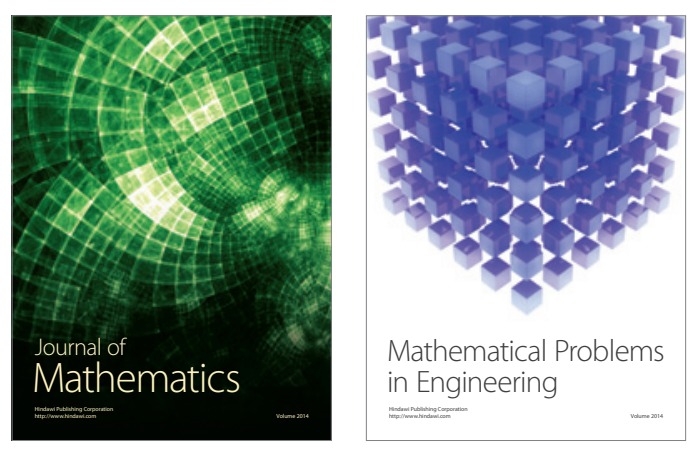

Mathematical Problems in Engineering
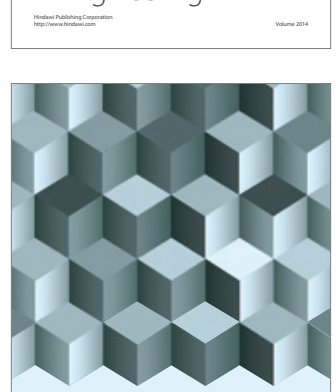

Journal of

Function Spaces
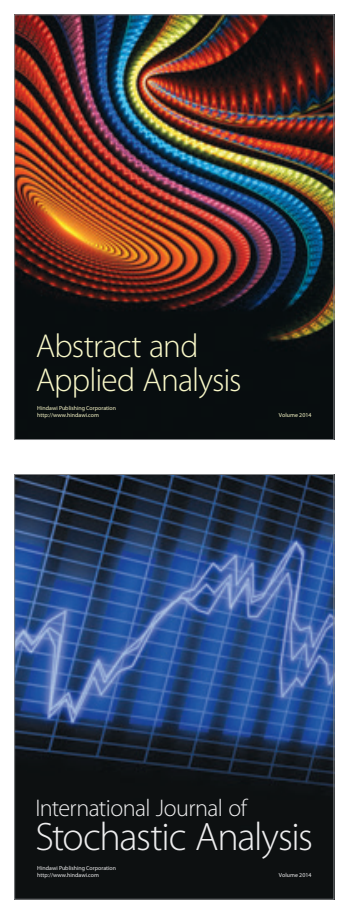

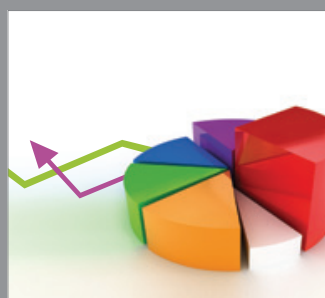

ournal of

Probability and Statistics

Promensencen
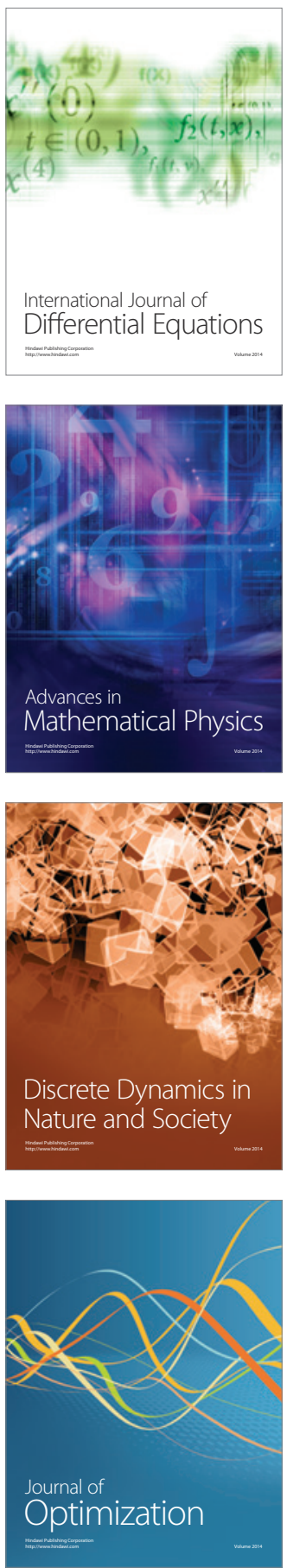